\title{
ON RELATIVE RATIONAL CHAIN CONNECTEDNESS OF THREEFOLDS WITH ANTI-BIG CANONICAL DIVISORS IN POSITIVE CHARACTERISTICS
}

\author{
YUAN WANG \\ ABSTRACT. In this paper we prove two results about the rational chain connectedness \\ for klt threefolds with anti-big canonical divisors in the relative setting.
}

\section{INTRODUCTION}

It is widely recognized that the geometry of a higher-dimensional variety is closely related to the geometry of rational curves on it. A classical result in the early 90s by Campana ([Cam92]) and Kollár-Miyaoka-Mori ([KMM92]) says that smooth Fano varieties are rationally connected in characteristic zero and are rationally chain connected in positive characteristics. This was later generalized by Zhang ([Zha06]) and HaconMcKernan ([HM07]) in characteristic zero. More recently using the minimal model program by Hacon-Xu ([HX15]) and Birkar ([Bir13]), Gongyo-Li-Patakfalvi-Schwede-TanakaZong $\left(\left[\mathrm{GLP}^{+} 15\right]\right)$ proved that projective globally $F$-regular threefolds in characteristic $\geq 11$ are rationally chain connected and this was later generalized to log Fano type threefold by Gongyo-Nakamura-Tanaka ([GNT15]).

The main result in [HM07] is as follows.

Theorem 1.1. [HM07, Theorem 1.2] Let $(X, \Delta)$ be a $\log$ pair, and let $f: X \rightarrow S$ be a proper morphism such that $-K_{X}$ is relatively big and $-\left(K_{X}+\Delta\right)$ is relatively semiample. Let $g: Y \rightarrow X$ be any birational morphism. Then the connected components of every fiber of $f \circ g$ are rationally chain connected modulo the inverse image of the locus of log canonical singularities of $(X, \Delta)$.

In this paper we prove a theorem similar to Theorem 1.1 for morphisms from a klt threefold to a variety of dimension $\geq 1$. More precisely, we have

Theorem A (Theorem 3.1). Let $X$ be a normal $\mathbb{Q}$-factorial threefold over an algebraically closed field $k$ of characteristic $\geq 7$ and $(X, D)$ a klt pair. Let $f: X \rightarrow Z$ be a proper morphism such that $f_{*} \mathcal{O}_{X}=\mathcal{O}_{Z}, \operatorname{dim}(Z)=1$ or $2, Z$ is klt, $-K_{X}$ is relatively big,

2010 Mathematics Subject Classification. Primary 14M22; Secondary 14E30.

Key words and phrases. Rational chain connectedness, positive characteristic, minimal model program, weak positivity, canonical bundle formula.

The author was supported in part by the FRG grant DMS-\#1265261. 
$-\left(K_{X}+D\right)$ is relatively semi-ample and $\left(X_{z}, D_{z}\right)$ is klt for general $z \in Z$. Let $g: Y \rightarrow X$ be any birational morphism. Then the connected components of every fiber of $f \circ g$ are rationally chain connected.

Motivated by Theorem A, we construct a global version of rational chain connectedness for threefolds.

Theorem B (Theorem 5.1). Let $X$ be a projective threefold over an algebraically closed field $k$ of characteristic $p>0, f: X \rightarrow Y$ a projective surjective morphism from $X$ to a projective variety $Y$ such that $f_{*} \mathcal{O}_{X}=\mathcal{O}_{Y}$. Let $D$ be an effective $\mathbb{Q}$-divisor, and $X_{\bar{\eta}}$ the geometric generic fiber of $f$. Assume that the following conditions hold.

(1) $(X, D)$ is $k l t,-K_{X}$ is big and $f$-ample, $K_{X}+D \sim_{\mathbb{Q}} 0$ and the general fibers of $f$ are smooth.

(2) $p>\frac{2}{\delta}$, where $\delta$ is the minmum non-zero coefficient of $D$.

(3) $D=E+f^{*} L$ where $E$ is an effective $\mathbb{Q}$-Cartier divisor such that $p \nmid \operatorname{ind}(E)$, $\left(X_{\bar{\eta}},\left.E\right|_{X_{\bar{\eta}}}\right)$ is globally $F$-split, and $L$ is a big $\mathbb{Q}$-divisor on $Y$.

(4) $\operatorname{dim}(Y)=1$ or 2 .

Then $X$ is rationally chain connected.

Here ind $(E)$ means the Cartier index of $E$.

The main ingredients of the proofs of Theorem A and Theorem B are the minimal model program constructed in [HX15], [Bir13] and $\left[\mathrm{GLP}^{+} 15\right]$; some facts, especially Theorem 2.1, in $\left[\mathrm{GLP}^{+} 15\right]$; some positivity results by Patakfalvi ([Pat14]) and Ejiri ([Eji15]); a canonical bundle formula constructed in Section 4 in the spirit of the paper [PS09] by Prokhorov and Shokurov. Note that the condition (3) in Theorem B is used in order to apply the result [Eji15, Theorem 1.1] of Ejiri to deduce that $-K_{Y}$ is big, and to apply Theorem 4.3 when $\operatorname{dim} Y=2$. This creates enough rational curves on $Y$. Note that by [Eji15, Example 3.4], $\left(X_{\bar{\eta}},\left.E\right|_{X_{\bar{\eta}}}\right)$ being globally $F$-split is equivalent to $S^{0}\left(X_{\bar{\eta}},\left.E\right|_{X_{\bar{\eta}}}, \mathcal{O}_{X_{\bar{\eta}}}\right)=H^{0}\left(X_{\bar{\eta}}, \mathcal{O}_{X_{\bar{\eta}}}\right)$.

Note that although the proof is independent, Theorem A can be implied by Theorem 4.1 of the paper [GNT15], which was put on arXiv before this paper. The proof of [GNT15, Theorem 4.1] relies on the minimal model program in dimension 3 in positive characteristic, which is only established in characteristic $\geq 7$ so far. On the other hand, Theorem B covers some cases in characteristic $<7$. In particular it does not rely on the minimal model program and is not implied by [GNT15, Theorem 4.1].

Acknowledgements. The author would like to express his gratitude to Christopher Hacon for suggesting this direction of research and a lot of valuable suggestions, comments, support and encouragement. He would like to thank Karl Schwede for answering many questions about $F$-singularities. He also thanks Omprokash Das, Honglu Fan and Zsolt Patakfalvi for helpful discussions. Finally he would like to thank the referee for many useful suggestions. 


\section{Preliminaries}

We work over an algebraically closed field $k$ of characteristic $p>0$.

\subsection{Preliminaries on rational connected varieties and the minimal model pro- gram.}

Definition 2.1. For a variety $X$ and a $\mathbb{Q}$-Weil divisor on $X$ such that $K_{X}+\Delta$ is $\mathbb{Q}$-Cartier. Let $f: Y \rightarrow X$ be a $\log$ resolution of $(X, \Delta)$ and we write

$$
K_{Y}=f^{*}\left(K_{X}+\Delta\right)+\sum_{i} a_{i} E_{i}
$$

where $E_{i}$ is a prime divisor. We say that $(X, \Delta)$ is

- sub Kawamata log terminal (sub-klt for short) if $a_{i}>-1$ for any $i$.

- Kawamata log terminal (klt for short) if $a_{i}>-1$ for any $i$ and $\Delta \geq 0$.

- $\log$ canonical if $a_{i} \geq-1$ for any $i$ and $\Delta \geq 0$.

Definition 2.2. [Kol96, IV.3.2] Suppose that $X$ is a variety over $k$.

(1) We say that $X$ is rationally chain connected $(R C C)$ if there is a family of proper and connected algebraic curves $g: U \rightarrow Y$ whose geometric fibers have only rational components and there is a cycle morphism $u: U \rightarrow X$ such that $u^{(2)}: U \times_{Y} U \rightarrow$ $X \times_{k} X$ is dominant.

(2) We say that $X$ is rationally connected $(R C)$ if (1) holds and moreover the geometric fibers of $g$ in (1) are irreducible.

Proposition 2.3. Let $X$ be a $k l t \mathbb{Q}$-factorial threefold over an algebraically closed field $k$ and $\operatorname{char}(k) \geq 7$. Let $g: W \rightarrow X$ be a log resolution and assume that $K_{W}+E=g^{*} K_{X}+B$, where $E$ and $B$ are exceptional divisors and the coefficients in $E$ are all 1 . Then relative minimal model for $(W, E)$ over $X$ exists. Denote this process by

$$
W=W_{0} \stackrel{f_{0}}{\rightarrow} W_{1} \stackrel{f_{1}}{\rightarrow} \underset{f_{N-1}}{\rightarrow} W_{N}=W^{\prime} .
$$

Then we actually have $W^{\prime}=X$. Moreover if we have a morphism $h: X \rightarrow Y$ such that every fiber of $h$ is $R C C$, then every fiber of $h \circ g$ is $R C C$.

Proof. The existence of this minimal model program is by $\left[\mathrm{GLP}^{+} 15\right.$, Theorem 3.2]. So we have a morphism $g^{\prime}: W^{\prime} \rightarrow X$ and we want to show that $g^{\prime}$ is the identity. Denote the strict transform of $E$ by $E^{\prime}$, then $K_{W^{\prime}}+E^{\prime}=g^{\prime *} K_{X}+B^{\prime}$ for some exceptional $\mathbb{Q}$-divisor $B^{\prime}$. By construction of the minimal model program we know that $g^{\prime *} K_{X}+B^{\prime}$ is nef over $X$ which means that $B^{\prime}$ is $g^{\prime}$-nef and since $X$ is klt the support of $B^{\prime}$ is the whole exceptional locus of $g^{\prime}$. So we can get that $B^{\prime}=0$ by negativity lemma, and since $X$ is $\mathbb{Q}$-factorial we will get $W^{\prime}=X$.

The proof of the last statement follows the proof of $\left[\mathrm{GLP}^{+} 15\right.$, Proposition 3.6]. Without loss of generality we can do a base change and assume that the base field $k$ is uncountable. 
Define $F$ in the following way: if $f_{i}$ is a divisorial contraction, then let $E_{0}=E, E_{i+1}=$ $f_{i, *} E_{i}$ and $F$ an arbitrary component of $E_{i}$; if $f_{i}$ is a flip and $C$ is any flipping curve then let $F$ be a component of $E_{i}$ that contains $C$. Let $K_{F}+\Delta_{F}:=\left.\left(K_{W_{i}}+E_{i}-\frac{1}{n}\left(E_{i}-F\right)\right)\right|_{F}$ where $n \gg 0$. By assumption $K_{W_{i}}+E_{i}-\frac{1}{n}\left(E_{i}-F\right)$ is plt, then by adjunction $K_{F}+\Delta_{F}$ is klt, hence by [Tan14, Theorem 14.4] $F$ is $\mathbb{Q}$-factorial. We also know that $-\left(K_{W_{i}}+E_{i}\right)$ is $f_{i}$-ample by assumption, then $-\left(K_{F}+\Delta_{F}\right)$ is ample. Moreover by [Pro01, Corollary 2.2.8] the coefficients of $\Delta_{F}$ are in the standard set $\left\{1-\frac{1}{n} \mid n \in \mathbb{N}\right\}$. Let $\tilde{F}$ be the normalization of $F$. Then by [HX15, Theorem 3.1] we know that $\left(\tilde{F}, \Delta_{\tilde{F}}\right)$ is strongly $F$-regular and by [HX15, Theorem 4.1] $F$ is a normal surface.

Next we consider three cases.

Case 1: If $f_{i}$ is a divisorial contraction and the exceptional divisor is contracted to a point, then since $-\left(K_{F}+\Delta_{F}\right)$ is ample, by [Kaw94, Lemma 2.2] $F$ is a rational surface, in particular it is rationally connected.

Case 2: If $f_{i}$ is a divisorial contraction and the exceptional divisor is contracted to a curve, then let $p: F \rightarrow B$ be the Stein factorization of $\left.f_{i}\right|_{F}$. By assumption $-\left(K_{F}+\Delta_{F}\right)$ is $f_{i}$-ample, so it is $p$-ample. Then for a general fiber $D$ of $p$ we have

$$
\left(K_{F}+D\right) \cdot D=\left(K_{F}+\Delta_{F}+D-\Delta_{F}\right) \cdot D=\left(K_{F}+\Delta_{F}\right) \cdot D-\Delta_{F} \cdot D<0 .
$$

Here $D$ is reduced and irreducible by [Băd01, Theorem 7.1]. Hence by [Tan14, Theorem 5.3] $D \cong \mathbb{P}^{1}$. Therefore every component of every fiber of $f_{i}$ is a rational curve.

Case 3: If $f_{i}$ is a flip, then let $C$ be an arbitrary flipping curve. By assumption we have $\left(K_{F}+\Delta_{F}\right) \cdot C<0, C^{2}<0$ and $0 \leq \operatorname{coeff}_{C} \Delta_{F}<1$, so $\left(K_{F}+C\right) \cdot C<0$. Again by [Tan14, Theorem 5.3] $C \cong \mathbb{P}^{1}$.

We denote a fiber of $h$ over $y \in Y$ by $F_{X, y}$. There is a morphism from $W_{i}$ to $Y$ for every $i$, and we denote denote the fiber of this morphism over $y$ as $F_{W_{i}, y}$. Then there is a rational map $F_{W_{i}, y} \rightarrow F_{W_{i+1}, y}$. From the above Case 1-3 we see that compared to $F_{W_{i}, y}$, there are only rational curves or a rational surface generated in $F_{W_{i+1}, y}$. So the RCC-ness of $F_{W_{i+1}, y}$ implies the RCC-ness of $F_{W_{i}, y}$. By assumption $F_{X, y}$ is RCC, so $F_{W, y}$ is RCC.

Proposition 2.4. Let $X$ be a klt $\mathbb{Q}$-factorial threefold over an algebraically closed field $k$ and $\operatorname{char}(k) \geq 7$. Let $f: X \rightarrow Y$ be a morphism from $X$ to a normal surface $Y$. Suppose we run a $K_{X}$-minimal model program and it terminates at $g: X^{\prime} \rightarrow Y$. If every fiber of $g$ is $R C C$ then every fiber of $f$ is $R C C$.

Proof. This can be easily deduced from Proposition 2.3 by taking a common resolution of $X$ and $X^{\prime}$. The proof of $\left[\mathrm{GLP}^{+} 15\right.$, Proposition 3.6] works as well.

2.2. Preliminaries on $F$-singularities. In this article, for a proper variety $X$, a $\mathbb{Q}$ divisor $\Delta$ and the line bundle $M$ we will use the concepts of strongly F-regular, the non $F$-pure ideal $\sigma(X, \Delta)$ and $S^{0}(X, \sigma(X, \Delta) \otimes M)$. The definitions of these can be found 
in many papers related to $F$-singularities (e.g. [HX15]). For a pair $(X, \Delta)$ where $\Delta$ is a $\mathbb{Q}$-Cartier divisor we also follow the definition of globally F-split in [Eji15].

Lemma 2.5. Let $X$ be a surface, $D$ an effective $\mathbb{Q}$-divisor on $X, f: X \rightarrow C$ a morphism from $X$ to a smooth curve $C$, and $\left(X_{c}, D_{c}\right)$ is a strongly $F$-regular pair for general $c \in C$. Assume that $-K_{X}$ is big, $K_{X}+D \sim_{\mathbb{Q}} 0$, then $C \cong \mathbb{P}^{1}$.

Proof. By Kodaira's Lemma we can write $D \sim_{\mathbb{Q}} \epsilon f^{*} H+E$ where $H$ is an ample $\mathbb{Q}$-divisor on $C, 0<\epsilon \in \mathbb{Q}, E$ is an effective $\mathbb{Q}$-divisor on $X$ and $\left(X_{c}, E_{c}\right)$ is also strongly $F$-regular for general $c \in C$ (since $X_{c}$ is a curve). Suppose that $C$ is not isomorphic to $\mathbb{P}^{1}$. We know that $K_{X / C}+E \sim_{\mathbb{Q}} f^{*}\left(-K_{C}-\epsilon H\right)$ is $f$-nef and $K_{X_{c}}+E_{c}$ is semi-ample for general $c \in C$, so by [Pat14, Theorem 3.16], $K_{X / C}+E=K_{X}-f^{*} K_{C}+E$ is nef. Since we have assumed that $g(C)>0$ we have that $K_{X}+E$ is nef. However this is impossible since $K_{X}+E \sim_{\mathbb{Q}}-\epsilon f^{*} H$ where $H$ is ample and $\epsilon>0$.

2.3. Weak positivity. Let $Y$ be a non-singular projective variety, $\mathcal{F}$ a torsion-free coherent sheaf on $Y$. We take $i: \hat{Y} \rightarrow Y$ to be the biggest open subvariety such that $\left.\mathcal{F}\right|_{\hat{Y}}$ is locally free. Let $\hat{S}^{k}(\mathcal{F}):=i_{*} S^{k}\left(i^{*} \mathcal{F}\right)$.

Definition 2.6. [Vie83, Definition 1.2] We call $\mathcal{F}$ weakly positive, if there is an open subset $U \subseteq Y$ such that for every ample line bundle $\mathcal{H}$ on $Y$ and every positive number $\alpha$ there exists some positive number $\beta$ such that $\hat{S}^{\alpha \cdot \beta}(\mathcal{F}) \otimes \mathcal{H}^{\beta}$ is generated by global sections over $U$.

Lemma 2.7. Weakly positive line bundles are nef.

Proof. This easily follows from Definition 2.6.

\section{Relative Rational Chain CONNECTEDNESs}

In this section we prove the following

TheOREm 3.1. Let $X$ be a normal $\mathbb{Q}$-factorial threefold over an algebraically closed field $k$ of characteristic $\geq 7$ and $(X, D)$ a klt pair. Let $f: X \rightarrow Z$ be a proper morphism such that $f_{*} \mathcal{O}_{X}=\mathcal{O}_{Z}, \operatorname{dim}(Z)=1$ or $2, Z$ is klt, $-K_{X}$ is relatively big, $-\left(K_{X}+D\right)$ is relatively semi-ample and $\left(X_{z}, D_{z}\right)$ is klt for general $z \in Z$. Let $g: Y \rightarrow X$ be any birational morphism. Then the connected components of every fiber of $f \circ g$ are rationally chain connected.

Proof. First we observe that $\left(X_{z}, D_{z}\right)$ being klt implies that $X_{z}$ is normal (in particular reduced) and irreducible.

Next we prove that if every fiber of $f$ is RCC, then every fiber of $f \circ g$ is RCC. We take a $\log$ resolution of $Y$ and denote it by $p: Y^{\prime} \rightarrow Y$ and let $q=g \circ p$. If we have $K_{Y^{\prime}}=q^{*} K_{X}+\tilde{B}$ then $K_{Y^{\prime}}-\tilde{B}=q^{*} K_{X}$ and the coefficients of $-\tilde{B}$ are $<1$. Then we can 
add another effective divisor to make all the coefficients 1 , and we denote this divisor by $\tilde{E}$. Now we run a relative $\left(K_{Y^{\prime}}+\tilde{E}\right)-\mathrm{MMP}$ of $Y^{\prime}$ over $X$. By Proposition 2.3 we see that if every fiber of $f$ is RCC then every fiber of $f \circ g \circ p$ is RCC, hence every fiber of $f \circ g$ is RCC.

Therefore it suffices to show that every fiber of $f$ is RCC. We consider the cases of $\operatorname{dim}(Z)=2$ and $\operatorname{dim}(Z)=1$ respectively.

Case 1: $\operatorname{dim}(Z)=2$.

If $\operatorname{dim}(Z)=2$ then a general fiber of $f$ being normal and $-K_{X}$ being relatively big implies that a general fiber of $f$ is a smooth rational curve. Next we run a relative minimal model program over $Z$ and denote this process as

$$
X=X_{0} \stackrel{f_{0}}{\rightarrow} X_{1} \stackrel{f_{1}}{\rightarrow} \underset{f_{N-1}}{\rightarrow} \stackrel{-\rightarrow}{\rightarrow} X_{n}=X^{\prime} .
$$

Since $-K_{X}$ is relatively big we end up with a Mori fiber space $X^{\prime} \stackrel{h}{\rightarrow} Z^{\prime} \stackrel{p}{\rightarrow} Z$ where $Z^{\prime}$ is also a surface. Then the general fibers of $h$ are rational curves. Moreover since $p_{*} \mathcal{O}_{Z^{\prime}}=\mathcal{O}_{Z}$ we know that $p$ is birational.

Now we prove that $h$ is equidimensional. Suppose that this is not the case, then there is a fiber $\tilde{F}$ of $h$ over a point $\tilde{z} \in Z^{\prime}$ which contains a 2-dimensional irreducible component. If $\tilde{F}$ is reducible then let $\tilde{F}_{1}$ be a 2-dimensional component of $\tilde{F}$ and $\tilde{F}_{2}$ another component which intersects $\tilde{F}_{1}$. We can choose a curve $\tilde{C}_{2} \subseteq \tilde{F}_{2}$ such that $\tilde{F}_{1} \cdot \tilde{C}_{2}>0$. On the other hand if we take a general point $z^{\prime} \in Z^{\prime}$ then $h^{-1}\left(z^{\prime}\right)$ is an irreducible curve and $h^{-1}\left(z^{\prime}\right) \cdot \tilde{F}_{2}=0$. This is a contradiction to the fact that $\rho\left(X^{\prime} / Z^{\prime}\right)=1$. If $\tilde{F}$ is irreducible, by Bertini's Theorem we have a very ample divisor $H \subset X^{\prime}$ such that $H \cap \tilde{F}$ is an irreducible curve which we denote by $\tilde{C}$. We do the Stein factorization of $\left.h\right|_{H}$ and denote the process as

$$
H \stackrel{h_{1}}{\longrightarrow} Z^{\prime \prime} \stackrel{h_{2}}{\longrightarrow} Z^{\prime}
$$

then $h_{1}$ is birational and $\tilde{C}$ is an exceptional curve of $h_{1}$. After possibly replacing $Z^{\prime \prime}$ by its normalization we can assume that $Z^{\prime \prime}$ is normal. Now $\tilde{F} \cdot \tilde{C}$ is equal to $\tilde{C}^{2}$ viewed as the self-intersection of $\tilde{C}$ in $H$, so by the Negativity Lemma it is $<0$. On the other hand we can still take a general point $z^{\prime} \in Z^{\prime}$ as above such that $h^{-1}\left(z^{\prime}\right) \cdot \tilde{F}=0$. This is also a contradiction to the fact that $\rho\left(X^{\prime} / Z^{\prime}\right)=1$.

Since $h$ is equidimensional, by [Deb01, Lemma 3.7] the components of every fiber of $h$ are rational curves. Then by Proposition 2.4 every fiber of $f$ is RCC.

Case 2: $\operatorname{dim}(Z)=1$.

Without loss of generality we can do a base change and assume that the base field $k$ is uncountable. By passing to the normalization of $Z$ we can assume that $Z$ is smooth. Then since every closed point of $Z$ is a Cartier divisor, every fiber of $f$ is also Cartier, hence $f$ is equidimensional. 
We first show that the general fibers of $f$ are rationally chain connected. Let $F$ be a general fiber of $f$. Since we assume that $\left(F,\left.D\right|_{F}\right)$ is klt, by adjunction we know that

$$
\left.\left.K_{X}\right|_{F} \equiv_{\text {num }}\left(K_{X}+F\right)\right|_{F}=K_{F}+\operatorname{Diff}_{F}(0),
$$

where $\operatorname{Diff}_{F}(0) \geq 0$ (cf. [Kol92, Proposition-Definition 16.5]). So $-\left(K_{F}+\operatorname{Diff}_{F}(0)\right)$, hence $-K_{F}$, is big. Therefore $\kappa(F)=-\infty$ and $F$ is birationally ruled by classification of surfaces. To prove that the general fibers of $f$ are RCC it suffices to prove that $F$ is rational. By assumption $-\left(K_{F}+\left.D\right|_{F}\right)=-\left.\left(K_{X}+D\right)\right|_{F}$ is semiample, so there exists an effective $\mathbb{Q}$-divisor $H$ such that $H \sim_{\mathbb{Q}}-\left(K_{F}+\left.D\right|_{F}\right)$ and $\left(F,\left.D\right|_{F}+H\right)$ is klt. We define $\Delta:=\left.D\right|_{F}+H$. Let $\pi: F^{\prime} \rightarrow F$ be a minimal resolution of $\left(F, \operatorname{Diff}_{F}(0)\right)$, then $F^{\prime}$ maps to a ruled surface $F^{\prime \prime}$ over a smooth curve $B$ via a sequence of blow-downs and we denote the morphism by $\psi$. The situation is as follows.

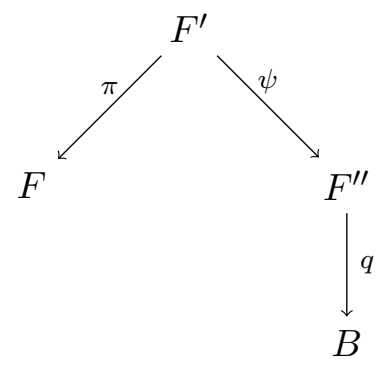

Since we have that $(F, \Delta)$ is klt, by [KM98, Throrem 4.7] $\pi$ and $\psi$ only contract $\mathbb{P}^{1} \mathrm{~s}$. So $F$ is RCC if and only if $F^{\prime \prime}$ is RCC. We define $\Delta^{\prime \prime}$ on $F^{\prime \prime}$ via the following

$$
K_{F^{\prime \prime}}+\Delta^{\prime \prime}=\psi_{*} \pi^{*}\left(K_{F}+\Delta\right) .
$$

Then $(F, \Delta)$ being klt implies that $\left(F^{\prime \prime}, \Delta^{\prime \prime}\right)$ is klt.

We denote a general fiber of $q$ by $R$. By construction $R \cong \mathbb{P}^{1}$, so we know that $\left(R,\left.\Delta^{\prime \prime}\right|_{R}\right)$ is klt and hence strongly $F$-regular. Then by applying Lemma 2.5 on $F^{\prime \prime}$ we know that $B=\mathbb{P}^{1}$. So $F$ is rational. Therefore we have proven that the general fibers of $f$ are RCC.

Since we have assumed that the base field $k$ is uncountable, by [Kol96, Ch. IV Corollary 3.5.2] we know that every fiber of $f$ is RCC.

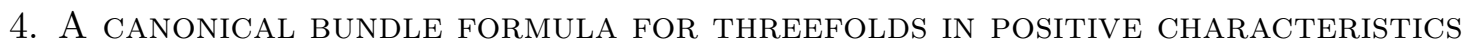

In this section following the idea of the proof of [PS09] we construct a canonical bundle formula in characteristic $p$ for a morphism from a threefold to a surface, whose general fibers are $\mathbb{P}^{1}$. There are similar constructions in [CTX13, 6.7] and [DH15, Theorem 4.8].

Let $\overline{\mathcal{M}}_{0, n}$ be the moduli space of $n$-pointed stable curves of genus $0, f_{0, n}: \overline{\mathcal{U}}_{0, n} \rightarrow \overline{\mathcal{M}}_{0, n}$ the universal family, and $\mathcal{P}_{1}, \mathcal{P}_{2}, \ldots, \mathcal{P}_{n}$ the sections of $f_{0, n}$ which correspond to the marked points. Let $d_{j}(j=1,2, \ldots, n)$ be the rational numbers such that $0<d_{j} \leq 1$ for all $j$, $\sum_{j} d_{j}=2$ and $\mathcal{D}=\sum_{j} d_{j} \mathcal{P}_{j}$. 
Lemma 4.1. [DH15, Lemma 4.6][Kaw97, Theorem 2]

(1) There exists a smooth projective variety $\mathcal{U}_{0, n}^{*}$, a $\mathbb{P}^{1}$-bundle $g_{0, n}: \mathcal{U}_{0, n}^{*} \rightarrow \overline{\mathcal{M}}_{0, n}$, and a sequence of blowups with smooth centers

$$
\overline{\mathcal{U}}_{0, n}=\mathcal{U}^{(1)} \stackrel{\sigma_{2}}{\longrightarrow} \mathcal{U}^{(2)} \stackrel{\sigma_{3}}{\longrightarrow} \ldots \stackrel{\sigma_{n-2}}{\longrightarrow} \mathcal{U}^{(n-2)}=\mathcal{U}_{0, n}^{*}
$$

(2) Let $\sigma: \overline{\mathcal{U}_{0, n}} \rightarrow \mathcal{U}_{0, n}^{*}$ be the induced morphism, and $\mathcal{D}^{*}=\sigma_{*} \mathcal{D}$. Then $K_{\overline{\mathcal{U}}_{0, n}}+\mathcal{D}-$ $\sigma^{*}\left(K_{\mathcal{U}_{0, n}^{*}}+\mathcal{D}^{*}\right)$ is effective.

(3) There exists a semi-ample $\mathbb{Q}$-divisor $\mathcal{L}$ on $\overline{\mathcal{M}}_{0, n}$ such that

$$
K_{\mathcal{U}_{0, n}^{*}}+\mathcal{D}^{*} \sim_{\mathbb{Q}} g_{0, n}^{*}\left(K_{\overline{\mathcal{M}}_{0, n}}+\mathcal{L}\right) .
$$

Definition 4.2. Let $f: X \rightarrow Y$ be a surjective proper morphism between two normal varieties and $K_{X}+D \sim_{\mathbb{Q}} f^{*} L$, where $D$ is a boundary divisor on $X$ and $L$ is a $\mathbb{Q}$-Cartier $\mathbb{Q}$-divisor on $Y$. Let $(X, D)$ be log canonical near the generic fiber of $f$, i.e., $\left(f^{-1} U,\left.D\right|_{f^{-1} U}\right)$ is $\log$ canonical for some Zariski dense open subset $U \subseteq Y$. We define

$$
D_{\mathrm{div}}:=\sum\left(1-c_{Q}\right) Q
$$

where $Q \subset Z$ are prime Weil divisors on $Z$ and

$$
c_{Q}=\sup \left\{c \in \mathbb{R}:\left(X, D+c f^{*} Q\right) \text { is } \log \text { canonical over the generic point } \eta_{Q} \text { of } Q\right\} .
$$

Next we define

$$
D_{\text {mod }}:=L-K_{Y}-D_{\text {div }}
$$

so $K_{X}+D=f^{*}\left(K_{Y}+D_{\mathrm{div}}+D_{\bmod }\right)$.

TheOREM 4.3. Let $f: X \rightarrow Y$ be a proper surjective morphism, where $X$ is a normal threefold and $Y$ is a normal surface over an algebraically closed field $k$ of characteristic $p>0$. Assume that $Q=\sum_{i} Q_{i}$ is a divisor on $Y$ such that $f$ is smooth over $(Y-\operatorname{Supp}(Q))$ with fibers isomorphic to $\mathbb{P}^{1}$. Let $D=\sum_{i} d_{i} D_{i}$ be $a \mathbb{Q}$-divisor on $X$ where $d_{i}=0$ is allowed, which satisfies the following conditions:

(1) $(X, D \geq 0)$ is klt on a general fiber of $f$.

(2) Suppose $D=D^{h}+D^{v}$ where $D^{h}$ is the horizontal part and $D^{v}$ is the vertical part of $D$. Then $p=\operatorname{char}(k)>\frac{2}{\delta}$, where $\delta$ is the minimum non-zero coefficient of $D^{h}$.

(3) $K_{X}+D \sim_{\mathbb{Q}} f^{*}\left(K_{Y}+M\right)$ for some $\mathbb{Q}$-Cartier divisor $M$ on $Y$.

Then we have that $D_{\text {mod }}$ is $\mathbb{Q}$-linearly equivalent to an effective $\mathbb{Q}$-divisor. Here $D_{\bmod }$ is defined as in Definition 4.2. Moreover if $(X, D)$ is klt then there exists an effective $\mathbb{Q}$-divisor $\bar{D}_{\text {mod }}$ on $Y$ such that $\bar{D}_{\text {mod }} \sim_{\mathbb{Q}} D_{\text {mod }}$ and $\left(Y, D_{\text {div }}+\bar{D}_{\text {mod }}\right)$ is klt.

Proof. First we reduce the problem to the case where all components of $D^{h}$ are sections. Let $D_{i_{0}}$ be a horizontal component of $D$ and $D_{i_{0}} \rightarrow D_{i_{0}}^{b} \rightarrow Y$ be the Stein factorization of $\left.f\right|_{D_{i_{0}}}$. Let $Y^{\prime} \rightarrow D_{i_{0}}^{b}$ be the normalization of $D_{i_{0}}^{b}$, then $Y^{\prime} \rightarrow Y$ is a finite surjective 
morphism of normal surfaces. Let $X^{\prime}$ be the normalization of the component of $X \times_{Y} Y^{\prime}$ dominating $Y$.

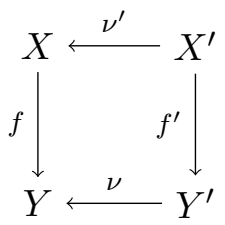

Let $m=\operatorname{deg}\left(\mu: \mathrm{Y}^{\prime} \rightarrow \mathrm{Y}\right)$ and $l$ be a general fiber of $f$. Then

$$
m=D_{i} \cdot l \leq \frac{1}{d_{i}}(D \cdot l)=\frac{1}{d_{i}}\left(-K_{X} \cdot l\right)=\frac{2}{d_{i}} \leq \frac{2}{\delta}<\operatorname{char}(k) .
$$

Therefore $\nu$ is a separable and tamely ramified morphism.

Let $D^{\prime}$ be the $\log$ pullback of $D$ under $\nu^{\prime}$, i.e.

$$
K_{X^{\prime}}+D^{\prime}=\nu^{\prime *}\left(K_{X}+D\right) \text {. }
$$

More precisely by [Kol92, 20.2] we have

$$
D^{\prime}=\sum_{i, j} d_{i j}^{\prime} D_{i j}^{\prime}, \quad \nu^{\prime}\left(D_{i j}^{\prime}\right)=D_{i} . \quad d_{i j}^{\prime}=1-\left(1-d_{i}\right) e_{i j}
$$

where $e_{i j}$ is the ramification indices along $D_{i j}^{\prime}$.

By construction $X$ dominates $Y$. Also, since $\nu$ is étale over a dense open subset of $Y$, say $\nu^{-1} U \rightarrow U$, and étale morphisms are stable under base change, $\left(f^{\prime} \circ \nu\right)^{-1} U \rightarrow f^{-1} U$ is étale. Thus the ramification locus $\Lambda$ of $\nu^{\prime}$ does not contain any horizontal divisor $f^{\prime}$, i.e. $f^{\prime}(\Lambda) \neq Y^{\prime}$. Therefore $D^{\prime}$ is a boundary near the generic fiber of $f^{\prime}$, i.e. $D^{\prime h}$ is effective. We observe that the coefficients of $D^{\prime h}$ can be computed by intersecting with a general fiber of $f^{\prime}: X^{\prime} \rightarrow Y^{\prime}$, hence they are equal to the coefficient of $D^{h} \subseteq X$. Thus the condition $p>\frac{2}{\delta}$ remains true for $D^{\prime}$ on $X^{\prime}$.

After finitely many such base changes we get a family $f^{\prime \prime}: X^{\prime \prime} \rightarrow Y^{\prime \prime}$, such that all of the horizontal components of $D^{\prime \prime}$ are rational sections of $f^{\prime \prime}$. Here $D^{\prime \prime}$ is the log pullback of $D$ via the induced finite morphism $\alpha: X^{\prime \prime} \rightarrow X$, i.e. $K_{X^{\prime \prime}}+D^{\prime \prime}=\alpha^{*}\left(K_{X}+D\right)$.

By construction of $\overline{\mathcal{M}}_{0, n}$ there is a generically finite rational map $Y^{\prime \prime} \rightarrow \overline{\mathcal{M}}_{0, n}$. Let $\beta_{0}: \tilde{Y} \rightarrow Y^{\prime \prime}$ be a morphism that resolves the indeterminacies of $Y^{\prime \prime} \rightarrow \overline{\mathcal{M}}_{0, n}$ and $\tilde{X}$ the normalization of $X^{\prime \prime} \times_{Y^{\prime \prime}} \tilde{Y}$. We have a morphism $\tilde{Y} \rightarrow \overline{\mathcal{M}}_{0, n}$ and let $\hat{X}=\tilde{Y} \times \overline{\mathcal{M}}_{0, n} \overline{\mathcal{U}}_{0, n}$. Let $X^{\sharp}$ be a common resolution of $\tilde{X}$ and $\hat{X}$. We have the following diagram: 


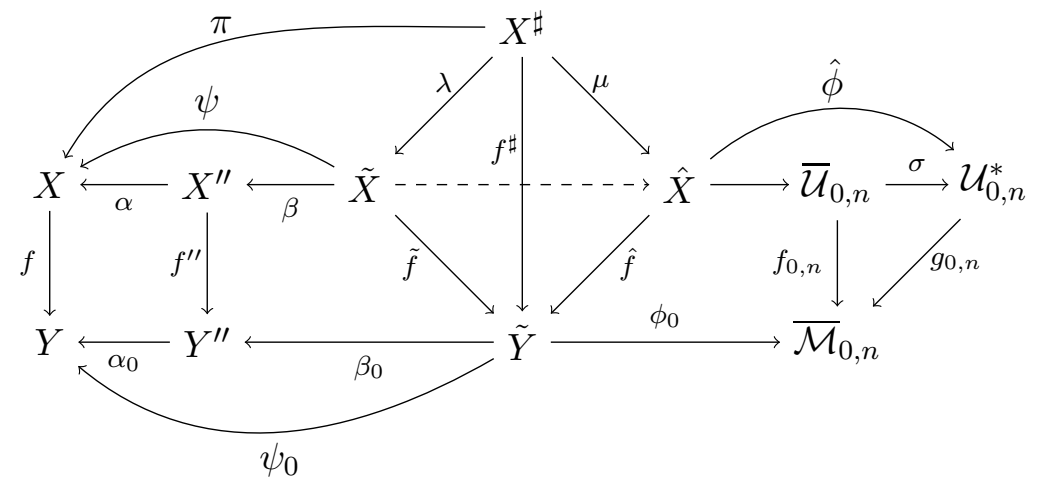

Let $D^{\sharp}$ and $\hat{D}$ be $\mathbb{Q}$-divisors on $X^{\sharp}$ and $\hat{X}$ respectively, defined by

$$
K_{X^{\sharp}}+D^{\sharp}=\pi^{*}\left(K_{X}+D\right)
$$

and

$$
K_{\hat{X}}+\hat{D}=\mu_{*}\left(K_{X^{\sharp}}+D^{\sharp}\right) .
$$

We also define $D_{\text {mod }}^{\prime \prime}$ and $D_{\text {div }}^{\prime \prime}$ on $Y^{\prime \prime}$ for $\left(X^{\prime \prime}, D^{\prime \prime}\right)$ as in Definition 4.2 , such that

$$
K_{X^{\prime \prime}}+D^{\prime \prime}=f^{\prime \prime *}\left(K_{Y^{\prime \prime}}+D_{\text {mod }}^{\prime \prime}+D_{\text {div }}^{\prime \prime}\right),
$$

and we define $\tilde{D}_{\text {mod }}$ and $\tilde{D}_{\text {div }}$ on $\tilde{Y}$ in a similar way. Since $K_{X^{\sharp}}+D^{\sharp}$ is the pullback of some $\mathbb{Q}$-divisor from the base $\tilde{Y}$ we get

$$
K_{X^{\sharp}}+D^{\sharp}=\mu^{*}\left(K_{\hat{X}}+\hat{D}\right) .
$$

Since $D_{\text {div }}$ does not depend on the birational modification of the family (see [PS09, Remark 7.3]), we will define it with respect to $\hat{f}: \hat{X} \rightarrow \tilde{Y}$.

Since $\hat{\phi}$ is generically finite and $\mathcal{D}^{*}$ is horizontal it follows that $\hat{\phi}^{*} \mathcal{D}^{*}$ is horizontal too. Since $\hat{D}^{h}$ is also horizontal one sees that

$$
\hat{D}^{h}=\hat{\phi}^{*} \mathcal{D}^{*}
$$

From the construction of $\sigma: \overline{\mathcal{U}}_{0, n} \rightarrow \mathcal{U}_{0, n}^{*}$ we see that $\left(F,\left.\mathcal{D}^{*}\right|_{F}\right)$ is log canonical for any fiber $F$ of $g_{0, n}: \mathcal{U}_{0, n}^{*} \rightarrow \overline{\mathcal{M}}_{0, n}$. Since the fibers of $\hat{f}: \hat{X} \rightarrow \tilde{Y}$ are isomorphic to the fiber of $g_{0, n},\left(\hat{F},\left.\hat{D}^{h}\right|_{\hat{F}}\right)$ is also log canonical, where $\hat{F}$ is any fiber of $\hat{f}$. Let $\hat{D}_{i}^{v}$ be a component of $\hat{D}^{v}$ and $\eta$ the generic point of $\hat{f}\left(\hat{D}_{i}^{v}\right)$. Then by inversion of adjunction we know that $\left(\hat{X}_{\eta},\left.\left(\hat{D}_{i}^{v}+\hat{D}^{h}\right)\right|_{\eta}\right)$ is log canonical. Since the fibers of $\hat{f}$ are reduced, the log canonical threshold of $\left(\hat{X}, \hat{D} ; \hat{D}_{i}^{v}\right)$ over the generic point of $\hat{D}_{i}^{v}$ is $\left(1-\operatorname{coeffi}_{\hat{D}_{i}^{v}} \hat{D}\right)$. Hence we get $\hat{D}^{v}=\hat{f}^{*} \tilde{D}_{\text {div }}$. Note that the coefficients of $\hat{D}^{v}$ can be $>1$. By definition of $\tilde{D}_{\text {mod }}$ we have

$$
K_{\hat{X}}+\hat{D}^{h} \sim_{\mathbb{Q}} \hat{f}^{*}\left(K_{\tilde{Y}}+\tilde{D}_{\text {mod }}\right) .
$$

Then we have

$$
K_{\hat{X}}+\hat{D}^{h}-f^{*}\left(K_{\tilde{Y}}+\phi_{0}^{*} \mathcal{L}\right)=K_{\hat{X} / \tilde{Y}}+\hat{D}^{h}-\hat{\phi}^{*} K_{\mathcal{U}_{0, n}^{*}} / \overline{\mathcal{M}}_{0, n}-\hat{\phi}^{*} \mathcal{D}^{*} \sim_{\mathbb{Q}} 0,
$$


where the first equality follows from (4.3) and Lemma 4.1 (3), and the second relation from (4.2) and [Liu02, Chapter 6 Theorem 4.9 (b) and Example 3.18].

Since $\hat{f}$ has connected fibers, by (4.3) and (4.4) and projection formula we get

$$
\tilde{D}_{\bmod } \sim_{\mathbb{Q}} \phi_{0}^{*} \mathcal{L}
$$

i.e. $\tilde{D}_{\text {mod }}$ is semi-ample.

Now since $\alpha_{0}: Y^{\prime \prime} \rightarrow Y$ is a composition of finite morphisms of degree strictly less than $\operatorname{char}(k)$ and $\beta_{0}$ is a birational morphism, by [Amb99, Theorem 3.2 and Example 3.1] we get

$$
K_{Y^{\prime \prime}}+D_{\mathrm{div}}^{\prime \prime} \sim_{\mathbb{Q}} \alpha_{0}^{*}\left(K_{Y}+D_{\mathrm{div}}\right)
$$

and

$$
K_{\tilde{Y}}+\tilde{D}_{\mathrm{div}} \sim_{\mathbb{Q}} \beta_{0}^{*}\left(K_{Y^{\prime \prime}}+D_{\mathrm{div}}^{\prime \prime}\right) .
$$

So $\alpha_{0}^{*} D_{\text {mod }} \sim_{\mathbb{Q}} D_{\text {mod }}^{\prime \prime}$, and $\beta_{0}^{*} D_{\text {mod }}^{\prime \prime} \sim_{\mathbb{Q}} \tilde{D}_{\text {mod }}$. By the projection formula we have

$$
D_{\text {mod }}^{\prime \prime} \sim_{\mathbb{Q}} \beta_{0, *} \tilde{D}_{\text {mod }}
$$

Then since $\alpha_{0}$ is finite,

$$
\psi_{0, *} \tilde{D}_{\text {mod }} \sim_{\mathbb{Q}} \alpha_{0, *} \beta_{0, *} \tilde{D}_{\text {mod }} \sim_{\mathbb{Q}} \alpha_{0, *} D_{\text {mod }}^{\prime \prime} \sim_{\mathbb{Q}} \alpha_{0, *} \alpha_{0}^{*} D_{\text {mod }} \sim_{\mathbb{Q}} D_{\text {mod }}
$$

Here we view the push-forward through $\alpha_{0}$ as push-forward of cycles. Therefore $D_{\text {mod }}$ is $\mathbb{Q}$-linearly equivalent to an effective divisor.

Next we prove the second statement. Since $\alpha$ is finite, by [Kol13, Corollary 2.42] we know that $\left(X^{\prime \prime}, D^{\prime \prime}\right)$ is klt, and as $\beta, \lambda$ and $\mu$ are birational we know that $(\hat{X}, \hat{D})$ is sub-klt, in particular $\hat{D}^{v}$ has coefficients $<1$. Since $\hat{f}$ is a $\mathbb{P}^{1}$ fibration and $\left(\tilde{Y}, \tilde{D}_{\text {div }}\right)$ is $\log$ smooth we have that $\left(\tilde{Y}, \tilde{D}_{\text {div }}\right)$ is sub-klt. By construction $\tilde{D}_{\text {mod }}$ is semi-ample, so by [Tan 15 , Theorem 1] we know that $\left(\tilde{Y}, \tilde{D}_{\text {div }}+\tilde{D}_{\text {mod }}\right)$ is sub-klt up to $\mathbb{Q}$-linear equivalence. Then $K_{Y^{\prime \prime}}+D_{\text {mod }}^{\prime \prime}+D_{\text {div }}^{\prime \prime} \sim_{\mathbb{Q}} \beta_{0, *}\left(K_{\tilde{Y}}+\tilde{D}_{\text {div }}+\tilde{D}_{\text {mod }}\right)$ is also sub-klt. Finally using [Kol13, Corollary 2.42] again and the fact that $D_{\text {mod }}+D_{\text {div }} \geq 0$ we get that $\left(Y, D_{\bmod }+D_{\text {div }}\right)$ is klt.

\section{Global Rational Chain connectedness}

In this section we prove the following theorem.

THeOREm 5.1. Let $X$ be a projective threefold over an algebraically closed field $k$ of characteristic $p>0, f: X \rightarrow Y$ a projective surjective morphism from $X$ to a projective variety $Y$ such that $f_{*} \mathcal{O}_{X}=\mathcal{O}_{Y}$. Let $D$ be an effective $\mathbb{Q}$-divisor, and $X_{\bar{\eta}}$ the geometric generic fiber of $f$. Assume that the following conditions hold.

(1) $(X, D)$ is $k l t,-K_{X}$ is big and $f$-ample, $K_{X}+D \sim_{\mathbb{Q}} 0$ and the general fibers of $f$ are smooth.

(2) $p>\frac{2}{\delta}$, where $\delta$ is the minmum non-zero coefficient of $D$. 
(3) $D=E+f^{*} L$ where $E$ is an effective $\mathbb{Q}$-Cartier divisor such that $p \nmid \operatorname{ind}(E)$, $\left(X_{\bar{\eta}},\left.E\right|_{X_{\bar{\eta}}}\right)$ is globally $F$-split, and $L$ is a big $\mathbb{Q}$-divisor on $Y$.

(4) $\operatorname{dim}(Y)=1$ or 2 .

Then $X$ is rationally chain connected.

REMARK 5.2. The smoothness of the general fibers of $f$ holds in characteristic $p \geq 11$ when $\operatorname{dim} Y=1$ by [Hir04, Theorem 5.1 (2)], and in characteristic $p \geq 5$ when $\operatorname{dim} Y=2$ by adjunction and a theorem of Tate (cf. [Lie13, Theorem 5.1]).

Proposition 5.3. Let $f: X \rightarrow Y$ be a projective surjective morphism between normal varieties with $f_{*} \mathcal{O}_{X}=\mathcal{O}_{Y}$. Assume that the following conditions hold.

(1) The general fibers of $f$ are isomorphic to $\mathbb{P}^{1}$.

(2) $Y$ is rationally chain connected.

Then $X$ is rationally chain connected.

Proof. The proof is essentially the same as $\left[\mathrm{GLP}^{+} 15\right.$, Lemma 3.12 and Proposition 3.13]. We take two general points $x_{1}, x_{2} \in X$ and let $y_{1}=f\left(x_{1}\right), y_{2}=f\left(x_{2}\right)$, by construction $f^{-1}\left(y_{1}\right) \cong f^{-1}\left(y_{2}\right) \cong \mathbb{P}^{1}$. By assumption $y_{1}$ and $y_{2}$ can be connected by a chain of rational curves, say $C_{1}, C_{2}, \ldots, C_{n}$. Let $\overline{C_{i}} \rightarrow C_{i}$ be the normalization for each $C_{i}, S_{i}:=f^{-1}\left(C_{i}\right)$, $\overline{S_{i}}:=S_{i} \times \overline{C_{i}} C_{i}$ and $g_{i}: \overline{S_{i}} \rightarrow S_{i}$ the induced morphisms. Now the morphism $\overline{S_{i}} \rightarrow \overline{C_{i}}$ is a flat projective morphism whose general fibers are $\mathbb{P}^{1}$, by [dJS03, Theorem] it has a section which we denote by $\tilde{C}_{i}$. Then $x_{1}$ and $x_{2}$ is connected by $f^{-1}\left(y_{1}\right), f^{-1}\left(y_{2}\right), g_{i}\left(\tilde{C}_{i}\right)$ and the fibers of $f$ over the intersection points of $\left\{C_{i}\right\}$, which is a union of rational curves by [Deb01, Lemma 3.7].

Proof of Theorem 5.1. We first prove the following lemma.

Lemma 5.4. Under the condition of Theorem 5.1, $-K_{Y}$ is big.

Proof. By assumption $m\left(K_{X_{\bar{\eta}}}+\left.E\right|_{X_{\bar{\eta}}}\right) \sim_{\mathbb{Q}} 0$ for sufficiently large and divisible $m$, in particular the $k(\bar{\eta})$-algebra $\bigoplus_{m \geq 0} H^{0}\left(\operatorname{am}\left(K_{X_{\bar{\eta}}}+\left.E\right|_{X_{\bar{\eta}}}\right)\right)$ is finitely generated. On the other hand since $\left(X_{\bar{\eta}},\left.E\right|_{X_{\bar{\eta}}}\right)$ is globally $F$-split we have that

$$
S^{0}\left(X_{\bar{\eta}}, \sigma\left(X_{\bar{\eta}},\left.E\right|_{X_{\bar{\eta}}}\right) \otimes \mathcal{O}_{X_{\bar{\eta}}}\left(m\left(K_{X_{\bar{\eta}}}+\left.E\right|_{X_{\bar{\eta}}}\right)\right)\right)=H^{0}\left(X_{\bar{\eta}}, \mathcal{O}_{X_{\bar{\eta}}}\left(m\left(K_{X_{\bar{\eta}}}+\left.E\right|_{X_{\bar{\eta}}}\right)\right)\right) .
$$

Here we would like to mention that for a line bundle $M$ and a $\mathbb{Q}$-Cartier divisor $\Delta$, the notation $S^{0}(X, \Delta, M)$ is the same as the standard notation $S^{0}(X, \sigma(X, \Delta) \otimes M$ ) (cf. [HX15, between Lemma 2.2 and Proposition 2.3]. Therefore by [Eji15, Theorem 1.1] we know that

$$
f_{*} \mathcal{O}_{X}\left(a m\left(K_{X / Y}+E\right)\right) \cong f_{*} \mathcal{O}_{X}\left(f^{*}\left(-a m\left(K_{Y}+L\right)\right)\right)=\mathcal{O}_{Y}\left(-a m\left(K_{Y}-L\right)\right)
$$

is weakly positive for $m \gg 0$. By Lemma $2.7,-K_{Y}-L$ is nef, so $-K_{Y}$ is big. 
Next we consider the following two cases.

Case 1: $Y$ is 1-dimensional.

After possibly taking the normalization of $Y$ we can assume that $Y$ is smooth. Then Lemma 5.4 implies that $g(Y)=0$, i.e. $Y \cong \mathbb{P}^{1}$. Let $F$ be a general fiber of $f$. By assumption $F$ is smooth and $K_{F}$ is anti-ample, hence $F$ is separably rationally connected. By [dJS03, Theorem] we know that $f$ has a section which we denote by $s$. Then $s(Y)$ is a rational curve in $X$ which dominates $Y$. Therefore we get that $X$ is rationally chain connected.

Case 2: $Y$ is 2-dimensional.

By assumption, a general fiber of $f$ is isomorphic to $\mathbb{P}^{1}$. Now by Lemma 5.4 we know that $-K_{Y}$ is big. On the other hand since $(X, D)$ is klt, by Theorem 4.3 there is a nonzero effective $\mathbb{Q}$-Cartier divisor $M$ on $Y$ such that $K_{Y}+M \sim_{\mathbb{Q}} 0$ and $(Y, M)$ is klt. Then by the proof of Case 2 of Theorem 3.1 we know that $Y$ is rational. Finally by Proposition 5.3 we get that $X$ is rationally chain connected.

\section{REFERENCES}

[Amb99] F. Ambro. The adjunction conjecture and its applications. Thesis (Ph.D.)-The Johns Hopkins University, 1999.

[Băd01] L. Bădescu. Algebraic Surfaces. Springer-Verlag, New York, 2001.

[Bir13] C. Birkar. Existence of flips and minimal model for 3-folds in char p. to appear in Ann. Sci. École Norm. Sup., 2013.

[Cam92] F. Campana. Connexité rationnelle de variétés de Fano. Ann. Sci. École Norm. Sup., 25(5):539545, 1992.

[Cor07] A. Corti, editor. Flips for 3-folds and 4-folds. 35. Oxford University Press, Oxford, 2007.

[CTX13] P. Cascini, H. Tanaka, and C. Xu. On base point freeness in positive characteristic. arXiv: 1305.3502, 2013.

[Deb01] O. Debarre. Higher-Dimensional Algebraic Geometry. Springer-Verlag, New York, 2001.

[DH15] O. Das and C. D. Hacon. On the Adjunction Formula for 3-folds in characteristic $p>5$. arXiv: 1505.05903, 2015.

[dJS03] A. J. de Jong and J. Starr. Every rationally connected variety over the function field of a curve has a rational point. Amer. J. Math., 125(3):567-580, 2003.

[Eji15] S. Ejiri. Weal positivity theorem and Frobenius stable canonical rings of geometric generic fibers. arXiv: 1508.00484v1, 2015.

$\left[\mathrm{GLP}^{+} 15\right]$ Y. Gongyo, Z. Li, Z Patakfalvi, K. Schwede, H. Tanaka, and H. Zong. On rational connectedness of globally F-regular threefolds. Adv. in Math., 280:47-78, 2015.

[GNT15] Y. Gongyo, Y. Nakamura, and H. Tanaka. Rationa points on log Fano threefolds over a finite field. arXiv: 1512.05003v1, 2015.

[Hir04] M. Hirokado. Deformations of rational double points and simple elliptic singularities in characteristic p. Osaka J. Math., 41(3):605-616, 2004.

[HM07] C. D. Hacon and J. McKernan. On Shokurov's rational connectedness conjecture. Duke Math. J., 138(1), 2007. 
[HP15] C. D. Hacon and Z. Patakfalvi. On the characterization of abelian varieties in characteristic $p>0$. arXiv: 1602.01791, 2015.

[HX15] C. D. Hacon and C. Xu. On the three dimensional minimal model program in positive characteristics. J. Amer. Math. Soc., 28(3):711-744, 2015.

[Kaw94] Y. Kawamata. Semistable minimal models of threefolds in positive or mixed characteristic. $J$. Algebraic Geom., 3(3):463-491, 1994.

[Kaw97] Y. Kawamata. Subadjunction of log canonical divisors for a subvariety of codimension 2. Contemp. Math., 207:79-88, 1997.

[KM98] J. Kollár and S. Mori. Birational Geometry of Algebraic Varieties. Cambridge University Press, Cambridge, 1998.

[KMM92] J. Kollár, Y. Miyaoka, and S. Mori. Rational connectedness and boundedness of Fano manifolds. J. Differential Geom., 36:765-779, 1992.

[Kol92] J. Kollár. Flips and abundance for algebraic threefolds. Société Mathématique de France, Paris, 1992.

[Kol96] J. Kollár. Rational Curves on Algebraic Varieties. Springer-Verlag, Berlin, 1996.

[Kol13] J. Kollár. Singularities of the minimal model program. Cambridge University Press, Cambridge, 2013.

[Lie13] C. Liedtke. Algebraic surfaces in positive characteristic. arXiv: 0912.4291v4, 2013.

[Liu02] Q. Liu. Algebraic geometry and arithmetic curves. Oxford University Press, Oxford, 2002.

[Pat14] Z. Patakfalvi. Semi-positivity in positive characteristics. Ann. Sci. École Norm Sup., 47(5):9911025, 2014.

[Pro01] Y. Prokhorov. Lectures on complements on log surfaces. Mathematical Society of Japan, Tokyo,, 2001.

[PS09] Y. Prokhorov and V. Shokurov. Towards the second main theorem on complements. J. Algebraic Geom., 18(1):151-199, 2009.

[Tan14] H. Tanaka. Minimal models and abundance for positive characteristic log surfaces. Nagoya Math. J., 216:1-70, 2014.

[Tan15] H. Tanaka. Semiample perturbations for log canonical varieties over an $F$-finite field containing an infinite perfect field. arXiv: 1503.01264v3, 2015.

[Vie83] E. Viehweg. Weak positivity and the additivity of the Kodaira dimension for certain fiber spaces. Adv. Stud. Pure Math., 1:329-353, 1983.

[Zha06] Q. Zhang. Rational connectedness of $\log \mathbb{Q}$-Fano varieties. J. Reine Angew. Math., 590:131-142, 2006.

Department of Mathematics

UNIVERSITY OF UTAH

155 South 1400 EAST

Salt Lake City, UT 84112-0090

USA

E-mail address: ywang@math.utah.edu 